\title{
SMOOTHLY SYMMETRIZABLE HYPERBOLIC SYSTEMS OF PARTIAL DIFFERENTIAL EQUATIONS
}

\author{
DALE CLARKE HERNQUIST
}

\begin{abstract}
.
In this paper we consider hyperbolic systems of linear, homogeneous, first order partial differential equations with variable coefficients. We will say that the Cauchy initial value problem for such a system is well posed if, for any sufficiently smooth initial data with compact support, there exists a unique classical solution which grows at most exponentially in time. Hyperbolicity alone does not guarantee the well-posedness of the Cauchy initial value problem for systems with variable coefficients. The Cauchy problem is well posed if the system is symmetric, or if it can be smoothly symmetrized (see [10]). The existence of smooth symmetrizers for systems whose roots have constant multiplicity has been proved (see [8]). We show that smooth symmetrizers exist for systems whose roots have multiplicities which vary between 1 and 2 , provided that the roots satisfy an algebraic condition. We then show 2 examples - one of a system whose roots satisfy the algebraic condition and the other of a system whose roots do not satisfy the algebraic conditions. We prove that the Cauchy problem for the second system is also well-posed, but that any symmetrizer of this system is not even twice differentiable. Thus smooth symmetrizability is sufficient, but not necessary for the well-posedness of the Cauchy problem.
\end{abstract}

\section{Introduction.}

Before proceeding it is necessary to state precisely the definitions of the terms used in the preceeding paragraph. A hyperbolic system of $N$ linear, homogeneous, first order partial differential equations in $n+1$ independent variables can be written in the form

$$
\frac{d u}{d t}=\sum_{i=1}^{n} A^{i}(x, t) \frac{d u}{d x_{i}} \stackrel{\text { der }}{=} P(x, t, d / d x) u,
$$

where $u$ is the $N$-vector $\left(u_{1}, \ldots, u_{N}\right)$ and for each $i=1, \ldots, n, A^{i}(x, t)$ is a smooth $N \times N$ matrix function of $(x, t)=\left(x_{1}, \ldots, x_{n}, t\right)$. With the system (1.1) we associate the $N \times N$ matrix $\widetilde{P}$, called the symbol of (1.1), defined by

$$
\widetilde{P}(x, t, \xi, \lambda)=\lambda I-P(x, t, \xi) .
$$

Received November 04, 1986. 
$\widetilde{P}$ is the $N \times N$ matrix whose elements are linear functions of $(\xi, \lambda)=\left(\xi_{1}, \ldots, \xi_{n}, \lambda\right)$ with coefficients which are functions of $(x, t)$. The characteristic equation of the system (1.1) is obtained by setting the determinant of the symbol equal to 0 . The roots of the characteristic equation are the eigenvalues of $P(x, t, \xi)$. For convenience, these roots are called the roots of the system. The system is hyperbolic if all of its roots are real.

The system (1.1) is called strongly hyperbolic if it is hyperbolic and uniformly diagonalizable. By this we mean that there exists a constant $K_{1}$, a nonsingular transformation $\Gamma=\Gamma(x, t, \xi)$, and a real diagonal matrix $\Lambda=\Lambda(x, t, \xi)$ such that

$$
\max _{(x, t, \xi)}\left\{|\Gamma(x, t, \xi)|,\left|\Gamma^{-1}(x, t, \xi)\right|\right\} \leqq K_{1}
$$

and

$$
\Lambda(x, t, \xi)=\left(\Gamma P \Gamma^{-1}\right)(x, t, \xi) .
$$

If the coefficients of the system are constant, $P(x, t, \xi)=P(\xi)$, then strong hyperbolicity implies the well-posedness of the Cauchy problem for all systems with $P(\xi)$ as principal part (see [9]).

If, in addition to being strongly hyperbolic, the system (1.1) can be smoothly symmetrized, then the Cauchy problem for (1.1) is well-posed (see $[10])$. We say that the system is symmetrizable if there exists a nondegenerate symmetric matrix $H=H(x, t, \xi)$ and a constant $K_{2}$ such that

$$
K_{2}^{-1} \leqq|H| \leqq K_{2},
$$

and

$$
(H P)(x, t, \xi)=(H P)^{T}(x, t, \xi)=\left(P^{T} H\right)(x, t, \xi)
$$

We say that it is smoothly symmetrizable if $H$ can be chosen to be a sufficiently smooth function of $x, \mathrm{t}$, and $\xi$. If $P$ is diagonalizable it is easy to show that any symmetrizer $H$ of $P$ can be written in the form

$$
H(x, t, \xi)=\left(\Gamma^{T} M \Gamma\right)(x t, \xi),
$$

where $\Gamma$ is as in (1.4). $M(x, t, \xi)$ is a diagonal matrix if the eigenvalues of $P(x, t, \xi)$ are distinct. In general $M(x, t, \xi)$ is a block diagonal matrix whose block size is determined by the multiplicity of the eigenvalues of $P(x, t, \xi)$. We see from (1.7) that if $\Gamma$ is a smooth function of $x, t$, and $\xi$, then the $H$ 
defined by (1.7) with $M$ taken to be the identity matrix is also a smooth function of $x, t$, and $\xi$.

\section{Description of the systems of interest.}

For which strongly hyperbolic systems do smooth symmetrizers exist? They exist for strictly hyperbolic systems, systems for which the eigenvalues of $P$ are distinct for all $(x, t, \xi)$. The corresponding $\Gamma$ defined by (1.4) is an analytic function of $x, t$, and $\xi$, so $H$ defined by (1.7) with $M$ taken to be the identity is analytic. However, recently it has been shown that strictly hyperbolic systems are extremely rare (see [2]). Over 20 years ago the existence of smooth symmetrizers for systems whose roots are not simple but have constant multiplicity was shown (see [8]). At that time there was interest in showing the existence of smooth symmetrizers for systems with multiple roots whose multiplicity varied. It is obvious from (1.7) that there is little hope of constructing smooth symmetrizers for such general systems. The best that might be done is to find smooth symmetrizers for systems whose roots have special properties. Special properties of the roots which might lead to smoothly symmetrizable systems were considered. However, no examples of systems with these special properties could be found.

In 1978, Fritz John published an example of a second order constant coefficient system of 3 equations in 4 independent variables, $\left(x_{1}, x_{2}, x_{3}, t\right)$, which is close to being strictly hyperbolic, but whose roots have varying multiplicity (see [3]). He showed that this system is strongly hyperbolic (see [4]). The system can be reduced to a first order homogeneous system of 7 equations. The reduction introduces a new root which is simple. The remaining roots of the reduced system have the same properties as those of the second order system. The first order system is also strongly hyperbolic (see [1]). Both systems are given in section 4. Here we will describe the properties of the roots of the first order system and generalize them to systems with constant and variable coefficients depending on an arbitrary number of independent variables. In the next section we will show that these systems can be smoothly symmetrized.

We begin by describing the geometry of the roots of the systems. The geometric conditions are easily visualized. They lead to an algebraic condition which will be used for more general systems. The eigenvalues of $P(\xi)$ are simple for all $\xi$ which do not lie on 2 lines through the origin of $\xi$-space, $\xi=0$. (Since the system is homogeneous, any 2 eigenvalues of $P(\xi)$ which are equal at $\xi_{0} \neq 0$ are equal for all $\xi$ on the line that passes through both $\xi_{0}$ and $\xi=0$.) For $\xi$ on these 2 lines, $P(\xi)$ has 2 sets of double eigenvalues and 3 simple eigenvalues. Each set of double eigenvalues is treated inde- 
pendently. Let $\lambda(\xi)$ and $\mu(\xi)$ denote 2 eigenvalues of $P(\xi)$ which are equal at $\xi_{0}$. Define the discriminant of $\lambda$ and $\mu$ by

$$
D(\xi)=(\lambda-\mu)^{2}(\xi)
$$

$D(\xi)$ is an analytic function of $\xi$ near $\xi_{0}$. At $\xi_{0}, D$ and its first derivatives are 0 . Thus we look at the matrix of the second derivatives of $D$, the Hessian of $D$, at $\xi_{0}$. Define

$$
\operatorname{Hess}\left(D\left(\xi_{0}\right)\right)=\left(\frac{\partial^{2} D}{\partial \xi_{i} \partial \xi_{j}}\left(\xi_{0}\right)\right)_{i, j=1,2,3}
$$

Hess $\left(D\left(\xi_{0}\right)\right)$ is positive semi-definite since our system is strongly hyperbolic. Its rank gives a measure of how rapidly the eigenvalues of $P(\xi)$ split apart for $\xi$ near $\xi_{0}$. Since $\lambda$ and $\mu$ are equal on a line through $\xi_{0}$, the rank of $\operatorname{Hess}\left(D\left(\xi_{0}\right)\right)$ must be either 1 or 2 . For our system the rank is 2 .

What are the essential properties of this system? It is important that the system is strongly hyperbolic, that the eigenvalues of $P(\xi)$ are simple for all $\xi$ except those lying on a finite number of lines through $\xi=0$, and that on these exceptional lines $P$ has only simple and double eigenvalues. The most important condition is that if $\lambda(\xi)$ and $\mu(\xi)$ are eigenvalues of $P(\xi)$ which are equal at $\xi_{0}$, then the rank of $\operatorname{Hess}\left(D\left(\xi_{0}\right)\right)$ as defined by $(2.1)$ and $(2.2)$ is 2 . It can be shown that this last algebraic condition quarantees that 2 previously mentioned conditions hold. First it quarantees that the system is strongly hyperbolic (see [1]). It also quarantees that $\lambda(\xi)$ and $\mu(\xi)$ are distint for all $\xi$ near $\xi_{0}$ which do not lie on the line through $\xi=0$ and $\xi_{0}$. This means that the requirement that there only be a finite number of lines on which $P(\xi)$ has double eigenvalues is extraneous. This is the algebraic condition mentioned earlier which is essential to the definition of the set of systems in which we are interested.

How do we generalize the above to systems with variable coefficients or to systems for which $n>3$. Suppose that $\lambda(x, t, \xi)$ and $\mu(x, t, \xi)$ are eigenvalues of $P(x, t, \xi)$ (both $x$ and $\xi$ are $n$-vectors, $n \geqq 3$ ) which coalesce when $(x, t, \xi)=\left(x_{0}, t_{0}, \xi_{0}\right)$. Then define

$$
D(x, t, \xi)=(\lambda-\mu)^{2}(x, t, \xi) .
$$

This is the obvious generalization of (2.1). We are interested in the Hessian. of $D(x, t, \xi)$ with respect to the $\xi$ variables only. Thus we generalize (2.2) to

$$
\operatorname{Hess}\left(D\left(x, t, \xi_{0}\right)\right)=\left(\frac{\partial^{2} D}{\partial \xi_{i} \partial \xi_{j}}\left(x, t, \xi_{0}\right)\right)_{i, j=1, \ldots, n} .
$$


Again we would like the rank of $\left.\operatorname{Hess}\left(D\left(x_{0}, t_{0}, \xi_{0}\right)\right)\right)$ to be maximal. We will prove later that the maximal rank is 2 . Thus the properties of the systems of the form (1.1) in which we are interested are as follows:

1. For all $(x, t, \xi)$ with $\xi \neq 0$ the eigenvalues of $P(x, t, \xi)$ are simple or double.

2. There exists a $\left(x_{0}, t_{0}, \xi_{0}\right), \xi_{0} \neq 0$, such that $P\left(x_{0}, t_{0}, \xi_{0}\right)$ has at least one pair of double eigenvalues.

3. If $\lambda(x, t, \xi)$ and $\mu(x, t, \xi)$ are eigenvalues of $P(x, t, \xi)$ which are equal when $(x, t, \xi)=\left(x_{0}, t_{0}, \xi_{0}\right), \xi_{0} \neq 0$, and if $D(x, t, \xi)$ is defined by $(2.3)$ and $\operatorname{Hess}\left(D\left(x_{0}, t_{0}, \xi_{0}\right)\right)$ is defined by (2.4), then the rank of $\operatorname{Hess}\left(D\left(x_{0}, t_{0}, \xi_{0}\right)\right)$ is 2 .

\section{Proof of the existence of smooth symmetrizers.}

It suffices to construct a smooth symmetrizer for $P(x, t, \xi)$ locally and to define it globally using a partition of unity. We have already noted that in a neighborhood of a point $\left(x_{0}, t_{0}, \xi_{0}\right)$ at which $P$ has distinct eigenvalues, the symmetrizer $H$ is defined by equation (1.7). Thus it suffices to construct $H$ in a neighborhood of a point $\left(x_{0}, t_{0}, \xi_{0}\right)$ at which $P\left(x_{0}, t_{0}, \xi_{0}\right)$ has at least one double eigenvalue. To do this we first transform $P$ into block diagonal form in a neighborhood of $\left(x_{0}, t_{0}, \xi_{0}\right)$. We define the block diagonal form in the following lemma.

Lemma 3.1. Assume that $P(x, t, \xi)$ is a diagonalizable $N \times N$ matrix with real eigenvalues and that $P\left(x_{0}, t_{0}, \xi_{0}\right)$ has $\varrho \geqq 1$ double eigenvalues and $N-2 \varrho$ simple eigenvalues. Then, in a neighborhood of $\left(x_{0}, t_{0}, \xi_{0}\right), P(x, t, \xi)$ is analytically similar to a matrix $B(x, t, \xi)$ which is the direct sum of $\varrho 2 \times 2$ matrices and a diagonal $(N-2 \varrho) \times(N-2 \varrho)$ matrix.

Proof. We 'need to define an analytic $T(x, t, \xi)$ for $(x, t, \xi)$ in a sufficiently small neighborhood of $\left(x_{0}, t_{0}, \xi_{0}\right)$ such that

$$
B(x, t, \xi)=\left(T P T^{-1}\right)(x, t, \xi)
$$

has the desired form. For simplicity we will define $T^{-1}$ instead of $T$.

Consider first the $N-2 \varrho$ simple eigenvalues of $P\left(x_{0}, t_{0}, \xi_{0}\right)$. Assume that the neighborhood of $\left(x_{0}, t_{0}, \xi_{0}\right)$ in which we are to define $B$ is sufficiently small that these eigenvalues are simple in the whole neighborhood. Then the last $N-2 \varrho$ columns of $T^{-1}$ are the eigenvectors of $P$ corresponding to these simple eigenvalues.

Assume that $\lambda(x, t, \xi)$ and $\mu(x, t, \xi)$ are eigenvalues of $P(x, t, \xi)$ which are equal when $(x, t, \xi)=\left(x_{0}, t_{0}, \xi_{0}\right)$. Assume further that the neighborhood in 
which we are to define $B$ is sufficiently small that $\lambda$ and $\mu$ are close to each other and separated from the other eigenvalues of $P$. Consider the spectral projection of $P$ corresponding to $\lambda$ and $\mu$. This projection is defined for $(x, t, \xi)$ near $\left(x_{0}, t_{0}, \xi_{0}\right)$ by

$$
\Pi(x, t, \xi)=\int_{\gamma}(P(x, t, \xi)-z I) d z
$$

where $\gamma$ separates $\lambda$ and $\mu$ from the remainder of the spectrum of $P$ (see [6]). Since $P$ is always diagonalizable, the range of $\Pi$ is 2 -dimensional in the neighborhood in which it is defined. The 2 independent vectors which span the range of $\Pi$ can be chosen to be analytic functions of $(x, t, \xi)$ in the neighborhood. The first $2 \varrho$ columns of $T^{-1}$ will consist of $\varrho$ pairs of these vectors.

What can we say about an arbitrary $2 \times 2$ block of the matrix $B$ defined in the lemma if $P(x, t, \xi)$ comes from a system of the form (1.1) which satisfies properties 1-3? Let $A(x, t, \xi)$ be an arbitrary $2 \times 2$ block of $B$. $A$ is only defined for $(x, t, \xi)$ near $\left(x_{0}, t_{0}, \xi_{0}\right)$. Since the eigenvalues of $A$ are also eigenvalues of $P$, they are real and are equal at $\left(x_{0}, t_{0}, \xi_{0}\right)$. $A$ is also uniformly diagonalizable. It is diagonal at $\left(x_{0}, t_{0}, \xi_{0}\right)$ since a diagonalizable $2 \times 2$ matrix with equal eigenvalues is diagonal. Let

$$
A(x, t, \xi)=\left(\begin{array}{ll}
a_{11} & a_{12} \\
a_{21} & a_{22}
\end{array}\right) .
$$

The discriminant $D(x, t, \xi)$ of the eigenvalues of $A$ is given by the equation

$$
D(x, t, \xi)=\left(a_{11}-a_{22}\right)^{2}+4 a_{12} a_{21} .
$$

Since the eigenvalues of $A$ are real, $D(x, t, \xi) \geqq 0$ for all $(x, t, \xi)$ for which it is defined. Once $D(x, t, \xi)$ is written in this form it is easy to see that the maximal rank of $\operatorname{Hess}\left(D\left(x_{0}, t_{0}, \xi_{0}\right)\right)$ is 2 .

Lemma 3.2. Assume that $P(x, t, \xi)$ is a diagonalizable $N \times N$ matrix with real eigenvalues which are simple or double for all $(x, t, \xi)$. If $\lambda(x, t, \xi)$ and $\mu(x, t, \xi)$ are eigenvalues of $P(x, t, \xi)$ which are equal when $(x, t, \xi)=\left(x_{0}, t_{0}, \xi_{0}\right)$, define $D(x, t, \xi)$ by equation $(2.3)$ and $\operatorname{Hess}\left(D\left(x_{0}, t_{0}, \xi_{0}\right)\right)$ by equation (2.4). The maximal rank of $\operatorname{Hess}\left(D\left(x_{0}, t_{0}, \xi_{0}\right)\right)$ is 2 .

Proof. We know from Lemma 3.1 that $P(x, t, \xi)$ can be written in block diagonal form near $(x, t, \xi)$. If the $2 \times 2$ block corresponding to $\lambda$ and $\mu$ is denoted by (3.3), $D$ is given by (3.4). Since $A\left(x_{0}, t_{0}, \xi\right)$ and $D\left(x_{0}, t_{0}, \xi\right)$ are analytic functions of $\xi$ near $\xi_{0}$, they can be written as a power series in 
$\xi-\xi_{0}$. The rank of $\left.\operatorname{Hess}\left(D\left(x_{0}, t_{0}, \xi_{0}\right)\right)\right)$ depends only on the terms of $D\left(x_{0}, t_{0}, \xi\right)$ which are quadratic in $\xi-\xi_{0}$. Since $A\left(x_{0}, t_{0}, \xi_{0}\right)$ is diagonal,

$$
\left(a_{11}-a_{22}\right)\left(x_{0}, t_{0}, \xi_{0}\right)=a_{12}\left(x_{0}, t_{0}, \xi_{0}\right)=0 .
$$

This means that the quadratic terms of $D\left(x_{0}, t_{0}, \xi\right)$ are determined solely by the linear terms of $a_{11}-a_{22}, a_{12}$, and $a_{21}$. The linear terms of these functions vanish on an $(n-1)$-dimensional plane through $\xi_{0}$. Thus the quadratic terms of $D\left(x_{0}, t_{0}, \xi\right)$ vanish on at least the intersection of $2(n-1)$-dimensional planes through the origin. The intersection of $2(n-1)$-dimensional planes which contain a common point is either $(n-1)$ or $(n-2)$-dimensional. Thus the quadratic terms of $D\left(x_{0}, t_{0}, \xi\right)$ must vanish on an $(n-2)$-dimensional surface. This implies that the maximal rank of $\left.\operatorname{Hess}\left(D\left(x_{0}, t_{0}, \xi_{0}\right)\right)\right)$ is 2 .

As soon as we construct a smooth symmetrizer for $A(x, t, \xi)$ we are done. The smooth symmetrizer of $B$ will just be the direct sum of the smooth symmetrizers of the $2 \times 2$ blocks and the $N-2 \varrho$ identity matrix. Denote the smooth symmetrizer of $B$ by $H_{B}$. If $T$ is the matrix in equation (3.1), then

$$
H(x, t, \xi)=\left(T^{T} H_{B} T\right)(x, t, \xi)
$$

is a smooth symmetrizer of $P(x, t, \xi)$ near $\left(x_{0}, t_{0}, \xi_{0}\right)$.

All that remains is to construct a smooth symmetrizer of $A(x, t, \xi)$. Now $A$ can be written as the sum of 3 symmetric matrices and a skew symmetric matrix. Define $\tau_{i}(x, t, \xi), i=0, \ldots, 3$ by

(3.6) $\tau_{0}=\frac{a_{11}+a_{22}}{2}, \quad \tau_{1}=\frac{a_{11}-a_{22}}{2}, \quad \tau_{2}=\frac{a_{12}+a_{21}}{2}, \quad \tau_{3}=\frac{a_{12}-a_{21}}{2}$.

The $\tau_{i}$ are analytic functions of $(x, t, \xi)$ near $\left(x_{0}, t_{0}, \xi_{0}\right)$. Then

$$
A=\tau_{0}\left(\begin{array}{ll}
1 & 0 \\
0 & 1
\end{array}\right)+\tau_{1}\left(\begin{array}{cc}
1 & 0 \\
0 & -1
\end{array}\right)+\tau_{2}\left(\begin{array}{ll}
0 & 1 \\
1 & 0
\end{array}\right)+\tau_{3}\left(\begin{array}{cc}
0 & 1 \\
-1 & 0
\end{array}\right)
$$

and

$$
D(x, t, \xi)=\tau_{1}^{2}+\tau_{2}^{2}-\tau_{3}^{2} .
$$

Since $D(x, t, \xi) \geqq 0$, equation (3.8) says that $\tau_{3}=0$ whenever $\tau_{1}=\tau_{2}=0$.

We would like to construct a nondegenerate symmetric matrix

$$
H=H(x, t, \xi)=\left(\begin{array}{ll}
h_{11} & h_{12} \\
h_{12} & h_{22}
\end{array}\right)
$$

which is an analytic function of $(x, t, \xi)$ near $\left(x_{0}, t_{0}, \xi_{0}\right)$ such that $(H A)(x, t, \xi)$ 
is symmetric. $H A$ will be symmetric if and only if

$$
2 h_{12} \tau_{1}+\left(h_{22}-h_{11}\right) \tau_{2}=\left(h_{11}+h_{22}\right) \tau_{3} \text {. }
$$

Note that $\tau_{0}$ does not appear in the equation, since $\tau_{0} H I$ is symmetric for any symmetric $H$. Thus it is sufficient to construct a symmetrizer for $A-\tau_{0} I$.

Equation (3.10) and the fact that $\tau_{3}=0$ whenever both $\tau_{1}$ and $\tau_{2}$ are 0 suggest that it might be possible and advantageous to change variables between two of the $\xi_{i}$ and $\left(\tau_{1}, \tau_{2}\right)$. Then we could define $H$ as a function of $\left(\tau_{1}, \tau_{2}\right)$ and the remaining $(n-2) \xi_{i}$. We can change coordinates between $\left(\tau_{1}, \tau_{2}\right)$ and, say for convenience, $\left(\xi_{1}, \xi_{2}\right)$ if $\tau_{1}\left(\dot{x}_{0}, t_{0}, \xi_{0}\right)=\tau_{2}\left(x_{0}, t_{0}, \xi_{0}\right)=0$ and if the Jacobian of the transformation,

$$
\left|\frac{\partial\left(\tau_{1}, \tau_{2}\right)}{\partial\left(\xi_{1}, \xi_{2}\right)}\right|\left(x_{0}, t_{0}, \xi_{0}\right)=\left(\frac{\partial \tau_{1}}{\partial \xi_{1}} \frac{\partial \tau_{2}}{\partial \xi_{2}}-\frac{\partial \tau_{1}}{\partial \xi_{2}} \frac{\partial \tau_{2}}{\partial \xi_{1}}\right)\left(x_{0}, t_{0}, \xi_{0}\right) \neq 0
$$

This inequality is a consequence of the assumption that the rank of $\operatorname{Hess}\left(D\left(x_{0}, t_{0}, \xi_{0}\right)\right)$ is 2 . By rearranging the $\xi_{i}$ if necessary, we may assume that

$$
\left(\frac{\partial^{2} D}{\partial \xi_{i} \partial \xi_{j}}\left(x_{0}, t_{0}, \xi_{0}\right)\right)_{i, j=1,2}
$$

is positive definite and, therefore, has a strictly positive determinant. A simple calculation, with $D$ defined by equation (3.8), shows that the determinant of (3.12) is

$$
\left(\left|\frac{\partial\left(\tau_{1}, \tau_{2}\right.}{\partial\left(\xi_{1}, \xi_{2}\right.}\right|^{2}-\left|\frac{\partial\left(\tau_{1}, \tau_{3}\right)}{\partial\left(\xi_{1}, \xi_{2}\right)}\right|^{2}-\left|\frac{\partial\left(\tau_{2}, \tau_{3}\right)}{\partial\left(\xi_{1}, \xi_{2}\right)}\right|^{2}\right)\left(x_{0}, t_{0}, \xi_{0}\right)
$$

The determinant can only be strictly positive if inequality (3.11) holds.

How does $\tau_{3}$ depend on $\tau=\left(\tau_{1}, \tau_{2}\right)$ ? To study this dependence, expand $\tau_{3}$ in a power series in $\tau$, letting $\xi=\left(\xi_{3}, \ldots, \xi_{n}\right)$.

$$
\tau_{3}(x, t, \tau, \xi)=c_{1}(x, t, \xi) \tau_{1}+c_{2}(x, t, \xi) \tau_{2}+\tilde{\tau},
$$

where $\tilde{\tau}$ is $O\left(\tau^{2}\right)$. If we denote the part of $A-\tau_{0} I$ which is linear in $\tau$ by $\bar{A}$ then

$$
\begin{aligned}
\tilde{A}(x, t, \tau, \xi) & =\tau_{1}\left(\begin{array}{cc}
1 & c_{1} \\
-c_{1} & -1
\end{array}\right)+\tau_{2}\left(\begin{array}{cc}
0 & 1+c_{2} \\
1-c_{2} & 0
\end{array}\right), \\
& \stackrel{\text { def }}{=} \tau_{1} A_{1}(x, t, \xi)+\tau_{2} A_{2}(x, t, \tilde{\xi}) .
\end{aligned}
$$


Now $\tilde{A}$ has distinct eigenvalues for $\tau \neq(0,0)$, and is thus diagonalizable. A theorem of Lax and Strang [9] shows how to construct a matrix $S(x, t, \xi)$ such that both $S A_{1} S^{-1}$ and $S A_{2} S^{-1}$ are symmetric. When both $A_{1}$ and $A_{2}$ are analytic functions of $(x, t, \xi)$, then so are $S$ and $S^{-1}$. Had we chosen the matrix $T$ in equation (3.1) of Lemma 1 to be $T S^{-1}$ instead of just $T$, the $\tilde{A}$ would have been symmetric to begin with. Assume that we chose $T$ properly and that $c_{1}=c_{2}=0$ in equations (3.14) and (3.15). Then $\tau_{3}$ is $O\left(\tau^{2}\right)$.

We would like to define $H$ as a power series in $\tau$ with coefficients which are analytic functions of $(x, t, \xi)$. If we can find the coefficients and show that the series converges, then $H$ will be an analytic symmetrizer of $A$. If we also write $\tau_{3}$ as a power series in $\tau$ with coefficients which are analytic functions of $(x, t, \xi)$, we can use equation (3.10) to solve for the coefficients of $H$ in terms of $\tau$ and the coefficients of $\tau_{3}$. We write

$$
\begin{aligned}
& H=\sum_{i, j=0}^{\infty} H^{i, j} \tau_{1}^{i} \tau_{2}^{j}, \\
& \tau_{3}=\sum_{i, j=0}^{\infty} \tau_{3}^{i, j} \tau_{1}^{i} \tau_{2}^{j} .
\end{aligned}
$$

Before substituting equations (3.16) and (3.17) into equation (3.10), we make some simplifying assumptions about $H$. We know that $\tau_{3}^{0,0}=\tau_{3}^{1,0}=\tau_{3}^{0.1}=0$. Thus we see from equation (3.10) that we may assume that $H^{0,0}=I$. We also assume that the trace of $H$ is constant. Since the trace of $H^{0,0}=I$ is 2 , we assume that $h_{11}+h_{22} \equiv 2$. With this assumption, equation (3.10) becomes

$$
h_{12} \tau_{1}+\frac{h_{22}-h_{11}}{2} \tau_{2}=\tau_{3} \text {. }
$$

Substituting (3.16) and (3.17) into (3.18) and equating coefficients of like powers of $\tau_{i} \tau_{j}$, we see that the $H^{i, j}, i+j=m$ are determined by the $\tau_{3}^{i, j}$, $i+j=m+1$. Consider the coefficients of the terms of order $m+1$ in equation (3.18). These coefficients must satisfy the following $m+2$ equations :

$$
\begin{aligned}
& 2 h_{12}^{m, 0}=2 \tau_{3}^{m+1,0} \\
& 2 h_{12}^{m-1,1}+\left(h_{22}-h_{11}\right)^{m, 0}=2 \tau_{3}^{m, 1} \\
& 2 h_{12}^{0, m}+\left(h_{22}-h_{11}\right)^{0, m-1}=2 \tau_{3}^{1, m} \\
& \left(h_{22}-h_{11}\right)^{0, m}=2 \tau_{3}^{0, m+1} \text {. }
\end{aligned}
$$

These are $m+2$ independent equations for $2 m+2$ variables. Thus the equations can be solved if the power series for $H$ converges. 
We will show that the power series converges for one fairly obvious choice of the $2 m$ extra variables for each $m$. For each $m$ choose

$$
h_{12}^{m-1,1}=h_{12}^{m-2,2}=\ldots=h_{12}^{1, m-1}=h_{12}^{0, m} .
$$

Solving for the remaining coefficients and summing the series, we see that

$$
\begin{aligned}
h_{12}\left(x, t, \tau_{1}, \tau_{2}, \xi\right) & =\frac{\tau_{3}\left(x, t, \tau_{1}, 0, \xi\right)}{\tau_{1}}, \\
\left(h_{22}-h_{11}\right)\left(x, t, \tau_{1}, \tau_{2}, \xi\right) & =2\left(\frac{\tau_{3}(x, t, \tau, \xi)-\tau_{3}\left(x, t, \tau_{1}, 0, \xi\right)}{\tau_{1}}\right)
\end{aligned}
$$

Since $\tau_{3}(x, t, \tau, \xi)$ is $O\left(\tau^{2}\right)$ the ,right hand sides of equations (3.20) and (3.21) are analytic functions. Since we assume earlier that the trace of $H=2$,

$$
H(x, t, \tau, \xi)=\left(\begin{array}{cc}
1+\frac{\tau_{3}\left(\tau_{1}, \tau_{2}\right)-\tau_{3}\left(\tau_{1}, 0\right)}{\tau_{1}} & \frac{\tau_{3}\left(\tau_{1}, 0\right)}{\tau_{1}} \\
\frac{\tau_{3}\left(\tau_{1}, 0\right)}{\tau_{1}} & 1-\frac{\tau_{3}\left(\tau_{1}, \tau_{2}\right)-\tau_{3}\left(\tau_{1}, 0\right)}{\tau_{1}}
\end{array}\right)
$$

$H(x, t, \xi)$ can be found by changing variables again. This concludes the proof of :

Theorem 3.3. Let $P(x, t, \xi)$ correspond to a hyperbolic system of partial differential equations of the form (1.1) and assume that $P(x, t, \xi)$ also satisfies properties 1-3. Then there exists a symmetric nondegenerate matrix $H(x, t, \xi)$ such that

$$
(H P)(x, t, \xi)=(H P)^{T}(x, t, \xi)=\left(P^{T} H\right)(x, t, \xi) .
$$

Moreover $H(x, t, \xi)$ is an infinitely smooth function of $(x, t, \xi)$.

\section{Examples.}

The example of a system of hyperbolic partial differential equations of the form (1.1) which satisfies properties $1-3$ of section 2 is derived from a system of 3 second order equations in 3 space variables. For $i=1,2,3$, the $i$ th equation of the second order system is

$$
\frac{\partial^{2} u_{i}}{\partial t^{2}}=\sigma_{i}(x, t) \Delta u_{i}+\left(1-\sigma_{i}\right) \frac{\partial}{\partial x_{i}}(\operatorname{div} u) .
$$

Here we assume that $0<\sigma_{1}(x, t)<\sigma_{2}(x, t)<\sigma_{3}(x, t)<1$ for all $(x, t)$. If the 
$\sigma_{i}$ were constant and equal, these would be the equations for elastic waves in a homogeneous, isotropic medium. System (4.1) with the $\sigma_{i}$ constant but satisfying the above inequalities was studied extensively by Fritz John (see [3], [4].) By changing dependent variables, (4.1) can be written as a first order system of 7 equations. Let

$$
\begin{array}{rlrl}
v_{1}=\frac{\partial u_{1}}{\partial t}, & v_{2} & =\frac{\partial u_{2}}{\partial t}, & v_{3}=\frac{\partial u_{3}}{\partial t}, \\
v_{4}=\frac{\partial u_{1}}{\partial x_{3}}-\frac{\partial u_{3}}{\partial x_{1}}, & v_{5}=\frac{\partial u_{2}}{\partial x_{1}}-\frac{\partial u_{1}}{\partial x_{2}}, & v_{6}=\frac{\partial u_{3}}{\partial x_{2}}-\frac{\partial u_{2}}{\partial x_{3}}, \\
v_{7} & =\operatorname{div} u . &
\end{array}
$$

If $v=\left(v_{1}, \ldots, v_{7}\right)$, then the system (4.1) can be written as

$$
\frac{\partial v}{\partial t}=P\left(x, t, \frac{d}{d x}\right) v
$$

where

$$
P(x, t, \xi)=\left(\begin{array}{rrrrrrr} 
& & & \sigma_{1} \xi_{3} & -\sigma_{1} \xi_{2} & & \xi_{1} \\
& & & & \sigma_{2} \xi_{1} & -\sigma_{2} \xi_{3} & \xi_{2} \\
\xi_{3} & & -\xi_{1} & & & & \\
-\xi_{2} & \xi_{1} & & & & & \\
& -\xi_{3} & \xi_{2} & & & & \\
\xi_{1} & \xi_{2} & \xi_{3} & & & &
\end{array}\right)
$$

The characteristic equation of system (4.3) is

$$
Q(x, t, \lambda, \xi)=\lambda\left(\lambda^{2}-|\xi|^{2}\right)\left(\sum_{r=1}^{3} \xi_{r}^{2}\left(\prod_{\substack{i=1 \\ i \neq r}}^{3}\left(\lambda^{2}-\sigma_{i}|\xi|^{2}\right)\right)\right)=0 .
$$

From equation (4.5) it is easy to see that the eigenvalues of $P(x, t, \xi)$ can be ordered as follows :

$$
\begin{gathered}
0=\lambda_{4}<\sqrt{\sigma_{1}}|\xi| \leqq \lambda_{5} \leqq \sqrt{\sigma_{2}}|\xi| \leqq \lambda_{6} \leqq \sqrt{\sigma_{3}}|\xi|<\lambda_{7}=|\xi| \\
\lambda_{1}=-\lambda_{7}, \quad \lambda_{2}=-\lambda_{6}, \quad \lambda_{3}=-\lambda_{5} .
\end{gathered}
$$

We see that the eigenvalues of $P(x, t, \xi)$ are never more than double and that when $P(x, t, \xi)$ has double eigenvalues, $\lambda_{2}=\lambda_{3}=-\sqrt{\sigma_{2}}$ and $\lambda_{5}=\lambda_{6}=\sqrt{\sigma_{2}}$. It can be shown that the eigenvalues are double on only 2 lines through the 
origin of $\xi$-space. These two lines intersect the unit sphere in $\xi$-space at the following 4 points, denoted by $\xi^{i}, i=1, \ldots, 4$.

$$
\begin{aligned}
\xi^{1} & =\left(\sqrt{\frac{\sigma_{2}-\sigma_{1}}{\sigma_{3}-\sigma_{1}}}, 0, \sqrt{\frac{\sigma_{3}-\sigma_{2}}{\sigma_{3}-\sigma_{1}}}\right), & \xi^{2} & =\left(\sqrt{\frac{\sigma_{1}-\sigma_{2}}{\sigma_{3}-\sigma_{1}}}, 0, \sqrt{\frac{\sigma_{3}-\sigma_{2}}{\sigma_{3}-\sigma_{1}}}\right) \\
\xi^{3} & =-\xi^{1} & \xi^{4} & =-\xi^{2} .
\end{aligned}
$$

The calculations to show that property 3 holds along these two exceptional lines are straightforward and tedious (see [1]). Thus they are omitted.

The example of a system of the form (1.1) which satisfies properties 1-3 came from altering a system of equations which correspond to a physical system. On the other hand the example of a system of the form (1.1) which satisfies properties 1 and 2, but not property 3 was found by trial and error. We looked at systems with constant coefficients because strong hyperbolicity implies the well-posedness of the Cauchy problem for these systems. We found a strongly hyperbolic system for which no symmetrizer of the system can be twice continuously differentiable.

Consider the system whose symbol is

$$
\tilde{P}(\lambda, \xi)=\lambda I-P(\lambda, \xi)=\lambda I-\left(\begin{array}{ccc}
0 & 0 & \xi_{1} \\
0 & 0 & -\xi_{2} \\
\xi_{1}+\xi_{2} & \xi_{1}-\xi_{2} & \xi_{3}
\end{array}\right) .
$$

The eigenvalues of $P(\lambda, \xi)$ are

$$
\begin{aligned}
& \lambda_{1}(\xi)=\frac{1}{2}\left(\xi_{3}-\sqrt{4 \xi_{1}^{2}+4 \xi_{2}^{2}+\xi_{3}^{2}}\right), \\
& \lambda_{2}(\xi)=0, \\
& \lambda_{3}(\xi)=\frac{1}{2}\left(\xi_{3}+\sqrt{4 \xi_{1}^{2}+4 \xi_{2}^{2}+\xi_{3}^{2}}\right) .
\end{aligned}
$$

These eigenvalues are real and distinct for all $\xi$ except those on the line $\xi=\left(0,0, \xi_{3}\right)$. For $\xi_{3}>0, \lambda_{1}(\xi)=\lambda_{2}(\xi)$, while for $\xi_{3}<0, \lambda_{2}(\xi)=\lambda_{3}(\xi)$. It is easy to see that $P(\xi)$ is diagonalizable for all $\xi$ and to construct the matrix of eigenvectors of $P(\xi)$. If the determinant of the matrix of eigenvectors of $P(\xi)$ is non-zero and finite, the system is strongly hyperbolic. A straightforward calculation shows that the value of the determinant is $1 \sqrt{2}$ for all $\xi$ (see [1]). Thus the Cauchy problem for system (4.6) is well-posed.

Does this system satisfy property 3 ? The eigenvalues of $P$ are analytic functions of $\xi$ away form $\xi=0$. Thus we can determine if the rank of Hessian 
of $D(\xi)$ is 2 by looking at the first order partial derivatives of $\left(\lambda_{i}-\lambda_{2}\right)(\xi)$ $=\lambda_{i}(\xi), i=1$ if $\xi_{3}>0$ and $i=3$ if $\xi_{3}<0$. The rank of the Hessian of $D(\xi)$ can not be 2 if the partials of $\lambda_{i}$ with respect to both $\xi_{1}$ and $\xi_{2}$ are zero at $\xi$. Both partials are zero, so property 3 does not hold.

TheOREm 4.1. If $P(\xi)$ is defined by (4.6) and if $H(\xi)$ is a symmetric twice continuously differentiable matrix such that

$$
(H P)(\xi)=(H P)^{T}(\xi)=\left(P^{T} H\right)(\xi),
$$

then $H(\tilde{\xi})$, where $\xi=\left(0,0, \xi_{3}\right)$, is not positive definite.

Proof. If equation (4.8) holds and if $H(\xi)$ is twice continuously differentiable, then for $i, j=1,2,3$

$$
\begin{aligned}
\left(H_{\xi_{i}} P+H P_{\xi_{i}}\right)(\xi) & =\left(P_{\xi_{i}}^{T} H+P^{T} H_{\xi_{i}}\right)(\xi), \\
\left(H_{\xi_{i} \xi_{j}} P+H_{\xi_{i}} P_{\xi_{j}}+H_{\xi_{j}} P_{\xi_{i}}\right)(\xi) & =\left(P_{\xi_{j}}^{T} H_{\xi_{i}}+P_{\xi_{i}}^{T} H_{\xi_{j}}+P^{T} H_{\xi_{i} \xi_{j}}\right)(\xi) .
\end{aligned}
$$

Note that equation (4.10) holds, because $P(\xi)$ is a linear function of $\xi$. Assume that $H(\xi)$ can be written as

$$
H(\xi)=\left(\begin{array}{lll}
h_{11} & h_{12} & h_{13} \\
h_{12} & h_{22} & h_{23} \\
h_{13} & h_{23} & h_{33}
\end{array}\right) .
$$

Equation (4.8) evaluated at $\xi$ implies that

$$
h_{13}(\xi)=h_{23}(\xi)=0 \text {. }
$$

Equations (4.9) evaluated at $\xi$ for $i=1,2$ imply

$$
\begin{aligned}
h_{33}(\xi) & =\frac{\partial h_{13}}{\partial \xi_{1}}(\xi)+h_{11}(\xi) \\
& =\frac{\partial h_{13}}{\partial \xi_{2}}(\xi)-h_{12}(\xi) \\
& =\frac{\partial h_{23}}{\partial \xi_{1}}(\xi)+h_{12}(\xi) \\
& =-\frac{\partial h_{23}}{\partial \xi_{2}}(\xi)+h_{22}(\xi) .
\end{aligned}
$$


Equations (4.10) evaluated at $(\xi)$ for $i=1,2$ implies

$$
\begin{gathered}
h_{33}(\xi)=h_{12}(\xi)=-h_{22}(\xi), \\
\frac{\partial h_{12}}{\partial \xi_{1}}+\frac{\partial h_{12}}{\partial \xi_{2}}=-\frac{\partial h_{13}}{\partial \xi_{1}}+\frac{\partial h_{13}}{\partial \xi_{2}} .
\end{gathered}
$$

Equations (4.13) and (4.14) together imply that

$$
\begin{aligned}
2 h_{11}(\xi) & =\left(-\frac{\partial h_{13}}{\partial \xi_{1}}+\frac{\partial h_{13}}{\partial \xi_{2}}\right)(\xi) \\
& =\left(-\frac{\partial h_{23}}{\partial \xi_{1}}-\frac{\partial h_{23}}{\partial \xi_{2}}\right)(\xi) .
\end{aligned}
$$

It is easy to see that equations (4.15) and (4.16) together imply that $h_{11}=0$. Then equation (4.14) implies that $h_{12}=h_{22}=0$. Thus $H(\xi)$ is degenerate and the theorem is proved.

Acknowledgements. I would like to thank Professor Fritz John and Professor Heinz Kreiss for directing my attention to this problem. The many stimulating conversations with them were extremely helpful in the completion of this work.

\section{BIBLIOGRAPHY}

1. D. Clarke, The Structure of the Set of Hyperbolic Systems of Partial Differential Equations, Ph. D. Thesis, New York University, 1981.

2. S. Friedland, J. W. Robbin and J. H. Sylvester, On the crossing rule, Com. Pure Appl. Math. 37 (1984), 19-37.

3. F. John, Algebraic conditions for hyperbolicity of systems of partial differential equations, Com. Pure Appl. Math. 31 (1978), 89-106.

4. F. John, Addendum to: Algebraic conditions for hyperbolicity of systems of partial differential equations, Com. Pure Appl. Math. 31 (1978), 787-793.

5. F. John, Partial Differential Equations, Third edition (Appl. Math. Sci. 6), Springer-Verlag, Berlin - New York, 1978.

6. T. Kato, A Short Introduction to Perturbation Theory for Linear Operators, pp. 72-84, Springer-Verlag, Berlin - New York, 1982.

7. H. O. Kreiss, Numerical Methods for Solving Time-Dependent Problems for Partial Differential Equations (Sém. Math: Supérieures 65), pp. 1-39, Presses de l'Université de Montréal, Que., 1978.

8. H. O. Kreiss, Über sachgemässe Cauchyprobleme, Math. Scand. 13 (1963), 109-128.

9. G. Strang, On strong hyperbolicity, J. Math. Kyoto Univ. 6 (1967), 397-417.

10. M. E. Taylor, Pseudodifferential Operators (Princeton Math. Ser. 34), Princeton University Press, Princeton, N.J. 1981, p. 78. 